\title{
TA Spectrum Summary
}

\section{Dmitri Ivanov*i}

University of Utah, Department of Physics \& Astronomy and High Energy Astrophysics Institute, Salt Lake City, Utah, USA

E-mail: dmiivanov@gmail.com

The Telescope Array (TA) covers an energy range from $4 \mathrm{PeV}$ to over $100 \mathrm{EeV}$. TA is a hybrid detector that uses air fluorescence detectors (FD) combined with a ground array. In May 2015, TA will have collected 7 years of data. The TA low energy extension (TALE), which sees cosmic rays down to $4 \mathrm{PeV}$, consists of additional fluorescence telescopes at one of the TA FD stations. An infill array of 400m spaced counters is being deployed. TALE has collected 1 year of data. We will present (1) the energy spectrum measured by TA surface detector, which provides the largest statistics measurement at the highest energies; (2) the TA FD mono and hybrid spectra which cover intermediate energies; and (3) we will show the first results of TALE, which include TALE monocular fluorescence and Čerenkov measurements and cover the lowest energies in the experiment.

The 34th International Cosmic Ray Conference,

30 July- 6 August, 2015

The Hague, The Netherlands

\footnotetext{
* Speaker.

${ }^{\dagger}$ For the Telescope Array Collaboration
} 


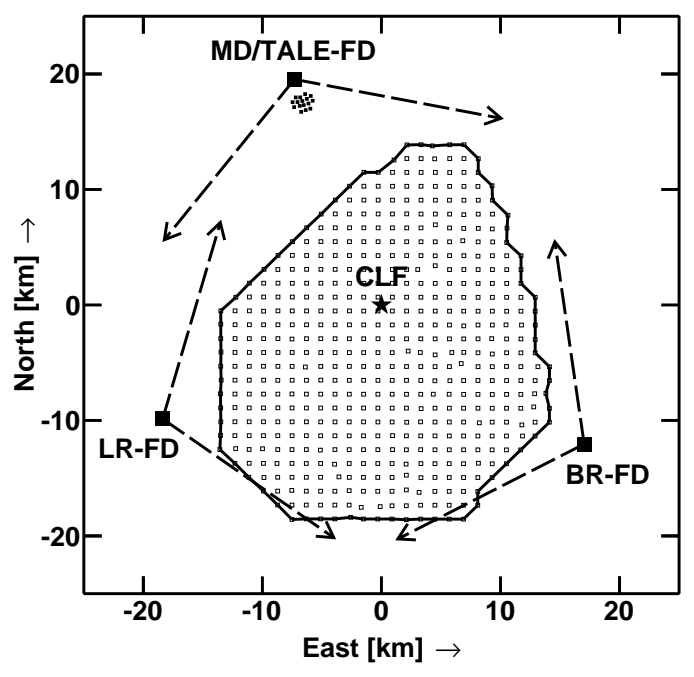

Figure 1: Layout of the TA/TALE detector using coordinates centered at the TA Central Laser Facility (CLF) labeled by the filled star. Open square boxes show the positions of the main TA SD counters, small filled square boxes correspond to the TALE infill array counters, and large filled squares with arrows show the 3 TA FD sites and their azimuthal viewing ranges.

\section{Introduction}

The Telescope Array(TA) experiment, located in Millard County, UT, USA, is measuring the ultra high energy cosmic rays since the year 2008 and it is the largest cosmic ray detector in the northern hemisphere. The TA has three fluorescence detector (FD) stations overlooking a surface detector array of 507 counters [14], each consisting of 2 layers of $3 \mathrm{~m}^{2} \times 1.2 \mathrm{~cm}$ scintillators. The counters are positioned on a $1200 \mathrm{~m}$ grid and span a $680 \mathrm{~m}^{2}$ area on the ground in total. The three TA FD stations are Black Rock Mesa (BR), Long Ridge (LR), and Middle Drum (MD). Both BR and LR have 10 telescopes each, with 256 pixels per telescope that use $10 \mathrm{MHz}$ FADC readout system, and each station covers $108^{\circ}$ in azimuth and 3 to $33^{\circ}$ degrees in elevation [15]. TA MD has 14 telescopes, with 256 pixels per telescope that use sample and hold electronics, and the station covers $112^{\circ}$ in azimuth and 3 to $31^{\circ}$ degrees in elevation [16]. The main TA detectors have been in operation since March 2008.

The TA low energy extension (TALE) consists of additional fluorescence telescopes added to the TA MD site and an infill array of same scintillation counters as used by the main TA, placed at distances 1.5 to $3 \mathrm{~km}$ away from the TA MD. There are currently 16 TALE infill counters on a $400 \mathrm{~m}$ square grid that are taking data since May 2013. TALE FD has 10 fluorescence telescopes with 256 pixels per telescope that use a 10MHz FADC readout system. TALE FD looks higher into the sky and extends the field of view of the TA MD from $31^{\circ}$ to $57^{\circ}$ in elevation. The TALE FD has been in full operation since September 2013. Figure 1 shows the layout of TA and TALE using coordinates centered at the TA Central Laser Facility (CLF).

The TA spectrum described here has been measured by 4 different analysis techniques. Since 
the FD duty cycle is limited by the daylight and weather, the SD stand-alone measurement gives the best answer for the spectrum at the highest energies: above $10^{19.0} \mathrm{eV}$ SD has good energy resolution and the exposure is uniform. The SD results starts at $10^{18.2} \mathrm{eV}$. For intermediate energies, we use TA FD monocular measurements, which cover energies $10^{17.2} \mathrm{eV}$ and above. For the energies between $10^{16.5}$ and $10^{18.4} \mathrm{eV}$, we use TALE FD because its mirrors are looking higher in elevation and it is possible to see lower energy showers by measuring fluorescence and Čerenkov light. Finally, for the lowest energy end of our result, $10^{15.6}$ to $10^{17.4} \mathrm{eV}$, we use showers that are seen in TALE FD and are reconstructed using only the Čerenkov light.

\section{TA SD}

Present work shows TA SD spectrum calculated using data from 2008/05/11 to 2015/05/11. Figure 4 shows SD resolution and exposure using solid lines. The TA SD energy spectrum calculation is done in 3 steps. First, AGASA [6] [7] reconstruction procedures are adjusted to fit the TA SD data. This is sufficient for reconstructing the event geometry and the lateral distribution, as shown in Figure 2.

Next, a detailed CORSIKA [1] QGSJET-II.3 [2] MC is generated. The thinning approximation [3] is used to speed up the process of generating CORSIKA showers and we use a method called dethinning [4] to restore the shower front information on the ground. The response of each TA SD unit to shower front particles is simulated using Geant4 [5]. The Monte-Carlo is reconstructed using same exact quality cuts and procedures as the data [17]. The TA SD MC uses proton composition and spectrum, as first measured by HiRes experiment [8], [9], and later confirmed by TA [10], [17]. The MC is then compared to the data to verify its validity. Figure 3 on the left left shows a typical data and MC comparison for the TA SD.

In the final step, an energy estimation table, shown in the middle of Figure 3, is derived from the $\mathrm{MC}$ and is used to reconstruct energies in both data and MC. The energies obtained from the energy estimation table are normalized by $1 / 1.27$ [17] to match the TA FD using well reconstructed events seen by both types of the TA detectors. This reduces the model dependence of the TA SD energy scale to that of the TA fluorescence detector. Plot on the right in Figure 3 shows a comparison of normalized SD event energies with those of BR, LR, and MD reconstructed in hybrid mode. In hybrid reconstruction, event geometries are constrained by both FD and SD, and event energies are reconstructed using shower atmospheric profiles seen by the FD.

\section{TA FD Mono}

The TA FD mono result presented here is from TA BR and LR detectors and covers dates from 2008/01/01 to 2014/12/28. Figure 4 shows the resolution and exposure of the TA BR/LR Mono spectrum analysis using dashed lines. TA FD reconstruction uses monocular geometry reconstruction, and fluorescence light produced in the atmosphere by charged particles of the shower. Details of TA FD mono analysis are discussed in this conference [11]. 

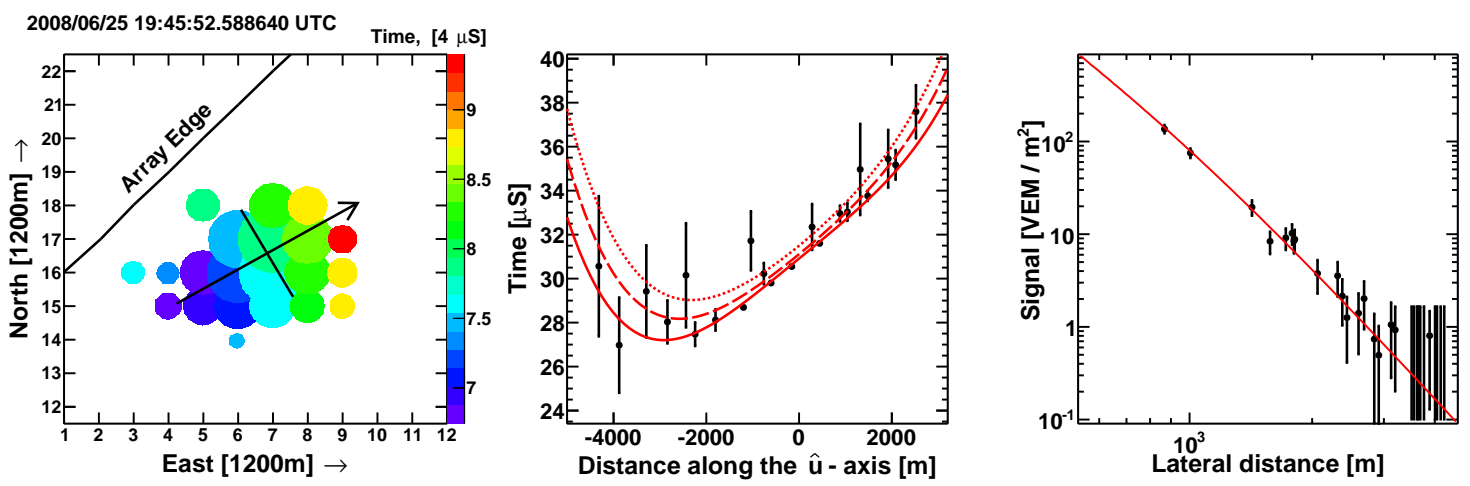

Figure 2: A typical high energy event seen by the TA SD. Left: each circle represents a counter, positioned at the center of the circle, the area of the circle is logarithmically proportional to the counter pulse height, and the counter time is denoted by the color. The arrow represents the projection of the shower axis onto the ground, denoted by $\hat{u}$, and it is bisected by the perpendicular line at the location of the shower core. Middle: counter time versus distance from the shower core along the $\hat{u}$ direction, which is the shower axis projected on the ground. Points with error bars are counter times, solid curve is the time expected by the fit for counters lying on the $\hat{u}$ axis, dashed and dotted lines are the fit expectation times for the counters that are correspondingly 1.5 and $2.0 \mathrm{~km}$ off the $\hat{u}$ axis. Right: Lateral distribution profile fit to the AGASA LDF. Vertical axis is the signal density in Vertical Equivalent Muon (VEM) per square meter units and horizontal axis is the lateral distance from the shower core. 1 VEM is $2.05 \mathrm{MeV}$ for the TA SD scintillator.
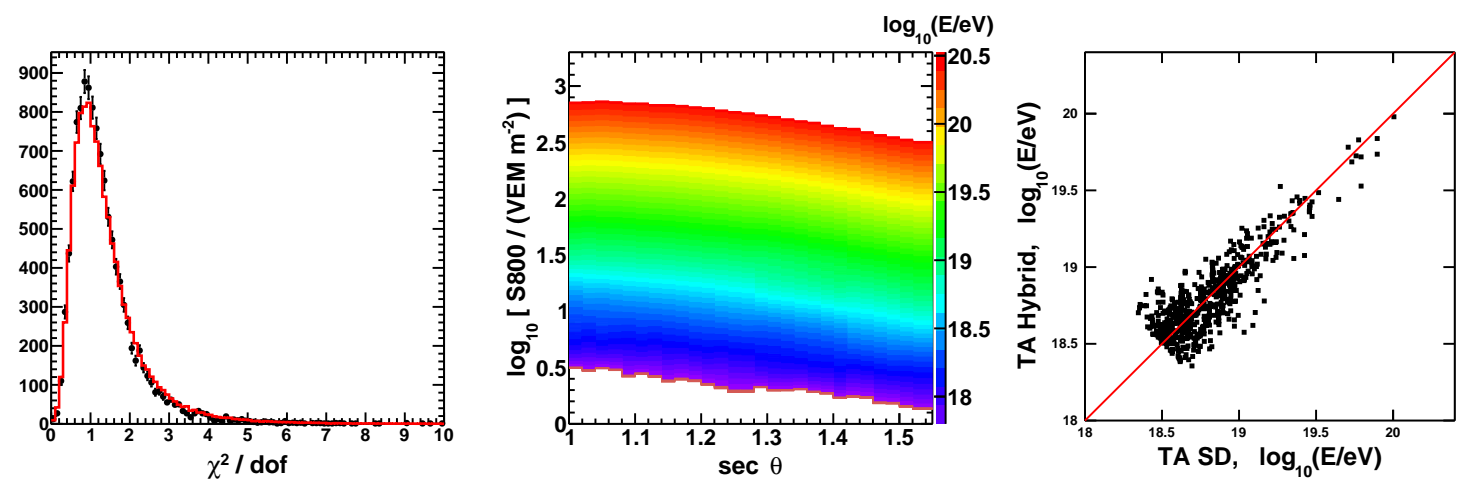

Figure 3: Right: TA SD data and MC comparison of the lateral distribution fit $\chi^{2}$ per degree of freedom. Points represent the data and solid line is the MC. Middle: Energy as function of reconstructed S800 and $\sec (\theta)$ made from the CORSIKA MC. Z-axis described by color represents the true (MC generated) values of energy. Right: TA SD reconstructed energies normalized by $1 / 1.27$ and compared to the TA Hybrid results of BR, LR, and MD simultaneously. Superimposed $45^{\circ}$ line shows no significant non-linearities.

\section{TALE FD Bridge}

The TALE bridge spectrum uses data collected in 2013/09/06 to 2014/01/09 period. Figure 4 shows the resolution and exposure of the TALE bridge spectrum analysis using dotted lines. The analysis uses geometry reconstructed in monocular mode and both fluorescence and Čerenkov components of light produced by particles of the shower. Details of the TALE bridge analysis are described in [13]. 

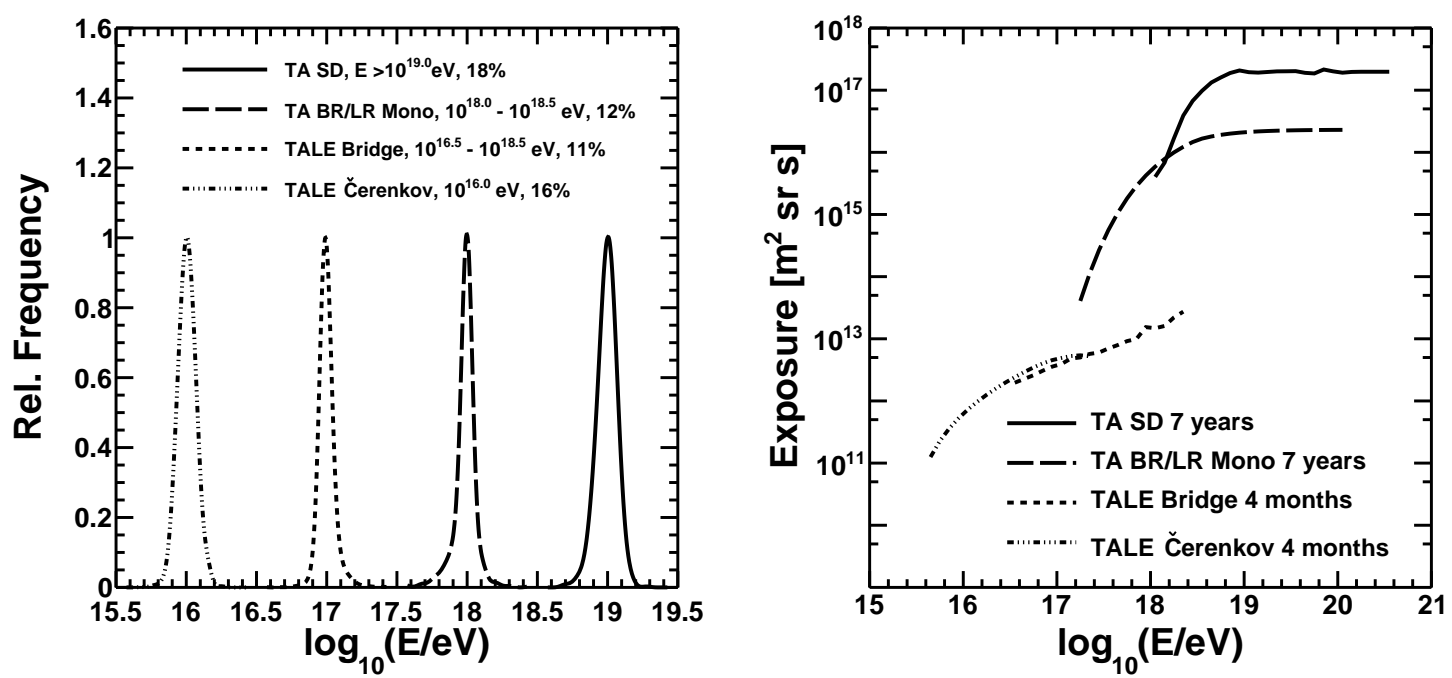

Figure 4: Right: Energy resolution for each TA/TALE analysis determined from the Monte-Carlo, displayed as histograms of $\log _{10}\left(E_{\mathrm{REC}} / E_{\mathrm{GEN}}\right)$ that are normalized so that the largest values in the bins are unity. $E_{\mathrm{GEN}}$ is the true energy generated in the $\mathrm{MC}$ and $E_{\mathrm{REC}}$ is the reconstructed energy obtained from processing MC using same analysis programs as for the data. The histograms have been shifted so that they are centered at the characteristic energies of each analysis. Left: Exposure for each analysis as function of energy using 0.1 width bins in $\log _{10}(E / e V)$.

\section{TALE FD Čerenkov}

The TALE Čerenkov spectrum uses data collected in 2013/09/06 to 2014/01/09 period. The analysis uses only the Čerenkov light that is produced by low energy events that occur close to the TALE FD site, at distances up to $1 \mathrm{~km}$. The analysis uses profile-constrained reconstruction of geometry, and Čerenkov light profile to determine the energy. The advantage of using profile constrained geometry in this case comes from the Čerenkov light profile shape being sensitive to the geometry of the shower track. The details of this analysis can be found in [12].

\section{Summary}

TA has measured spectrum over 4.7 orders in magnitude in energy. Figure 5 shows the result with superimposed fit to a single broken power law function to characterize the 4 spectral features seen in the TA spectrum. The GZK cutoff [18], [19] is seen at $E=10^{19.80 \pm 0.05} \mathrm{eV}$, with spectral indices of $-2.66 \pm 0.02$ before and $-4.65 \pm 0.6$ after the break. The ankle, explained by the pair production of cosmic microwave background photons in the vicinity of a proton primary [20], is seen at $10^{18.72 \pm 0.02} \mathrm{eV}$, with an index of $-3.226 \pm 0.007$ immediately before it.

Finally, TA/TALE sees two additional features at lower energies: a second knee and a low energy ankle. The second knee occurs at $10^{17.30 \pm 0.05} \mathrm{eV}$, with power law of $-2.94 \pm 0.02$ to the left of the feature, and the low energy ankle is seen at $10^{16.34 \pm 0.04} \mathrm{eV}$. The power law is $-3.132 \pm 0.001$ before the low energy ankle. These new results are preliminary, and the systematic uncertainties are currently being evaluated. 


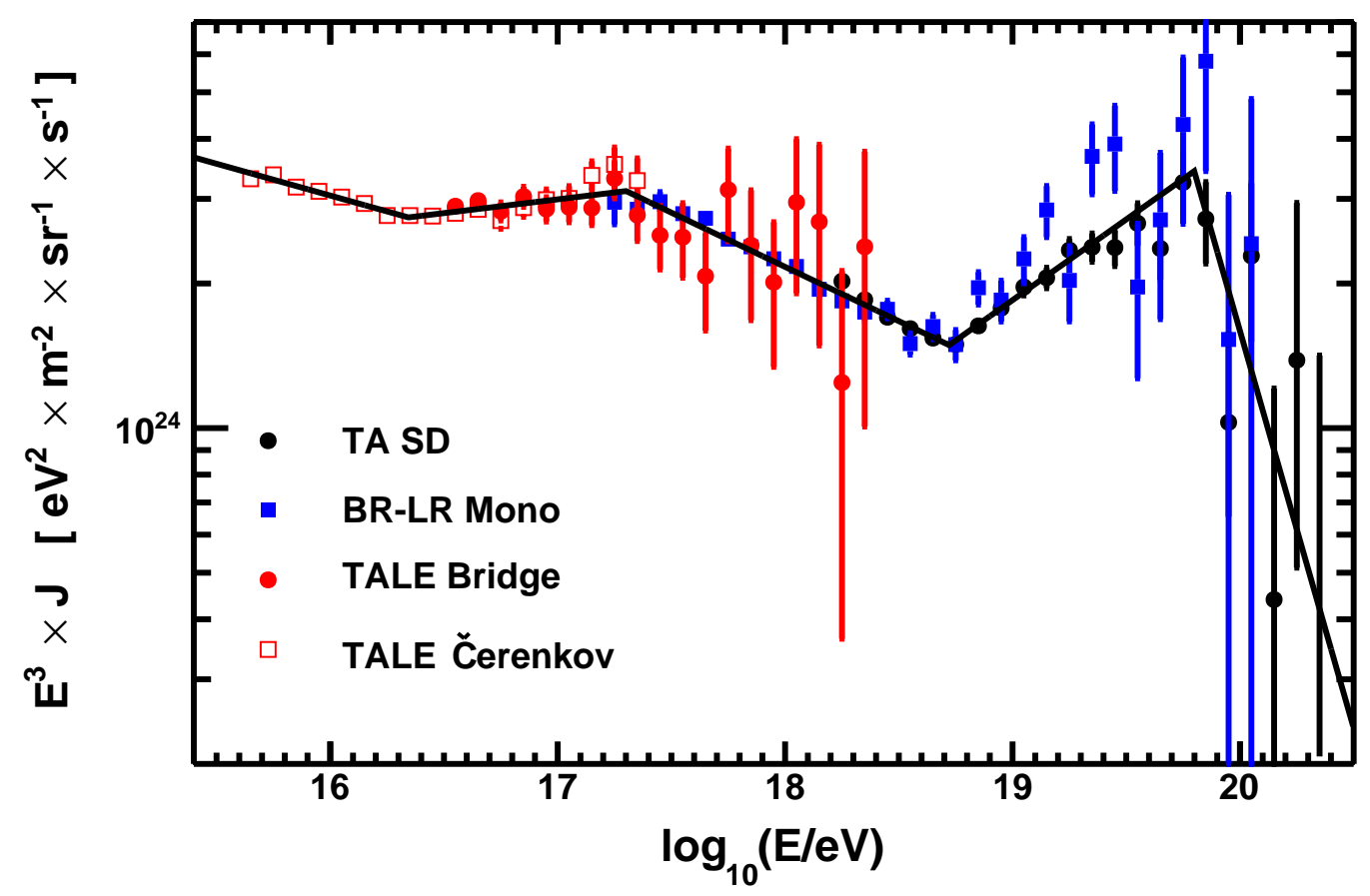

Figure 5: Energy spectrum measured by TA.

For the TA SD and BR/LR Mono, the systematic uncertainty is dominated by the energy scale, which is currently estimated $20 \%$ for TA. The uncertainty due to calculation of the exposure from Monte-Carlo is within 3\%.

\section{Acknowledgements}

The Telescope Array experiment is supported by the Japan Society for the Promotion of Science through Grants-in-Aid for Scientific Research on Specially Promoted Research (21000002) "Extreme Phenomena in the Universe Explored by Highest Energy Cosmic Rays" and for Scientific Research (19104006), and the Inter-University Research Program of the Institute for Cosmic Ray Research; by the U.S. National Science Foundation awards PHY-0307098, PHY-0601915, PHY-0649681, PHY-0703893, PHY-0758342, PHY-0848320, PHY-1069280, PHY-1069286, PHY1404495 and PHY-1404502; by the National Research Foundation of Korea (2007-0093860, R3210130, 2012R1A1A2008381, 2013004883); by the Russian Academy of Sciences, RFBR grants 11-02-01528a and 13-02-01311a (INR), IISN project No. 4.4502.13, and Belgian Science Policy under IUAP VII/37 (ULB). The foundations of Dr. Ezekiel R. and Edna Wattis Dumke, Willard L. Eccles, and George S. and Dolores Doré Eccles all helped with generous donations. The State of Utah supported the project through its Economic Development Board, and the University of Utah through the Office of the Vice President for Research. The experimental site became available through the cooperation of the Utah School and Institutional Trust Lands Administration (SITLA), 
U.S. Bureau of Land Management, and the U.S. Air Force. We also wish to thank the people and the officials of Millard County, Utah for their steadfast and warm support. We gratefully acknowledge the contributions from the technical staffs of our home institutions. An allocation of computer time from the Center for High Performance Computing at the University of Utah is gratefully acknowledged.

\section{References}

[1] J. Knapp and D. Heck, Nachr. Forsch. zentr. Karlsruhe 30 (1998) 27.

[2] S. Ostapchenko, Nucl. Phys. Proc. Suppl. 151 (2006) 143 [hep-ph/0412332].

[3] M. Kobal, Astropart. Phys. 15, (2001) 259

[4] B. T. Stokes, R. Cady, D. Ivanov, J. N. Matthews and G. B. Thomson, Astropart. Phys. 35 (2012) 759 [arXiv:1104.3182 [astro-ph.IM]].

[5] J. Allison, et al., IEEE Trans. Nucl. Sci. 53 (2006) 270

[6] K. Shinozaki and M. Teshima [AGASA Collaboration], Nucl. Phys. Proc. Suppl. 136, 18 (2004).

[7] M. Teshima et al., J. Phys. G 12 (1986) 1097.

[8] R. U. Abbasi et al. [HiRes Collaboration], Phys. Rev. Lett. 104 (2010) 161101 [arXiv:0910.4184 [astro-ph.HE]].

[9] R. U. Abbasi et al. [HiRes Collaboration], Phys. Rev. Lett. 100 (2008) 101101 [arXiv:astro-ph/0703099].

[10] R. U. Abbasi, M. Abe et al. [Telescope Array Collaboration], Astropart. Phys. 64 (2014) 49 [arXiv:1408.1726 [astro-ph.HE]].

[11] Toshihiro Fujii [Telescope Array Collaboration], "Energy Spectrum and Mass Composition of Ultra-High Energy Cosmic Rays Measured with the Telescope Array Fluorescence Detector Using a Monocular Analysis" Contribution ID 320, ICRC-2015, Hague, NL

[12] T. AbuZayyad [Telescope Array Collaboration], "Cosmic Rays Energy Spectrum observed by the TALE detector using Cerenkov light " Contribution ID 422, ICRC-2015, Hague, NL

[13] Z. Zundel [Telescope Array Collaboration], "Fluoresence Detection of Cosmic Ray Air Showers with the Telescope Array Low Energy Extension“ Contribution ID 445, ICRC-2015, Hague, NL

[14] T. Abu-Zayyad et al. [Telescope Array Collaboration], Nucl. Instrum. Meth. A 689 (2012) 87 [arXiv:1201.4964 [astro-ph.IM]].

[15] T. Abu-Zayyad et al. [Telescope Array Collaboration], Nucl. Instrum. Meth. A 609 (2009) 227

[16] T. Abu-Zayyad et al. [Telescope Array Collaboration], Astropart. Phys. 39-40 (2012) 109 [arXiv:1202.5141 [astro-ph.IM]].

[17] T. Abu-Zayyad et al. [Telescope Array Collaboration], Astrophys. J. 768 (2013) L1 [arXiv:1205.5067 [astro-ph.HE]].

[18] K. Greisen, Phys. Rev. Lett. 16 (1966) 748.

[19] G. T. Zatsepin and V. A. Kuzmin, limit of the spectrum of cosmic rays," JETP Lett. 4 (1966) 78 [Pisma Zh. Eksp. Teor. Fiz. 4 (1966) 114]. = ZFPRA,4,114;

[20] R. Aloisio, V. Berezinsky and S. Grigorieva, Astropart. Phys. 41 (2013) 94 [arXiv:1006.2484 [astro-ph.CO]]. 